Research on the chemical constitution of the blood in these patients may be a helpful line which $I$ intend to investigate further.

Class D.

This consists of cases in which operations in the throat had been badly performed, and which cleared up after thorough removal of adenoids.

\section{Class E.}

Cases in this class are included merely to show the material on which this communication is based.

\section{Gonclusions.}

The points which have appealed to me in reviewing these cases are:

1. That the presence of pus or muco-pus in the nose must suggest the possibility of sinus infection, although the presence of adenoids may produce the same picture.

2. That when dealing with infection of the maxillary antrum in children the possibility of ethmoid infection at an early age must be kept in mind.

3. That before deciding on any operative form of treatment of a child's nose or throat a careful history should be obtained. This mas form a useful guide to prognosis.

\section{PERSISTENT NASAL CATARRH IN CHILDREN.*}

DOUGLAS GUTHRIE, M.D., F.R.C.S.ED., AURAL SURGEON, ROYAL HOSPITAL FOR SICK CHILDREN, EDINBURGH.

Persistent nasal catarrh, or, in scientific language, chronic rhinitis, is a common disease in childhood, and its causes are numerous. The foremost cause is, of course, adenoids, and the adenoid growth is of two varieties. There is, first, tho large central tumour mass; and secondly, the type in which the lymphoid growth is spread as a sheet over the roof and posterior wall of the nasopharynx. It is this second variety which is more liable to cause nasal discharge, and the discharge may persist even after careful operation.

Another cause of nasal discharge in children is diphtheritic infection, and we have found it so common in the Children's Hospital that we take a swab from every case of nasal discharge. I mention this in passing in order to emphasize the importance of bacteriological examination.

As regards sinus suppuration, there is no doubt that the antra and ethmoids may become infected at an early agc, as is proved by the work of Dean and others. in America, and of Cleminson in London. My colleague Dr. Hall has followed up those researches and has kindly permitted me to quote his results, which are not yet published. He performed lavage of the antra in 85 cases from which adenoids and tonsils were to be removed. In 10 per cent. he found actual creamy pus in the antra, in 13 per cent. there was muco-pus, and in 27 per cent. the saline which he aspirated back into the syringe was turbid. The majority of such cases are curable by removal of adenoids, though a few cases may require operation on the antrum-namely, intranasal drainage.

The type of case which particularly interests us to-day is the little patient who suffers from persistent nasal discharge for which no apparent cause can be found. How frequently, for example, does one meet with children whose nasopharynges have been curetted on two or more occasions, without result!

Such patients suffer from nasal obstruction, as well as from nasal discharge, and $I$ venture to submit that it is the obstruction, rather than the discharge, which constitutes the real cause of all the trouble. In fact, the so-called "persistent nasal catarih" is caused by pyogenic infection in a narrow and poorly drained nasal cavity. In an obstructed nose secretions accumulate and readily become infected. The obstruction arises from arrested development of the nose and nasopharynx, caused by the adoption of a mouth-breathing habit at an early age. And how has this mouth-breathing habit originated? From a *A communication to the Section of Oto-Rhino-Laryngology at the
Annual Meeting of the British Medical Association, Fanchester, 1929 . succession of head colds during the first few years of life. The sequence of events is as follows: recurrent attacks of acute rhinitis, nasal obstruction and mouth-breathing, arrest of nasal development and eventually chronio rhinitis. The appearance of adenoids as an attempt to combat infection is a mere incident, though a fortunate incident if the adenoids are of the large tumour variety. Operation then effects a dramatic cure. But frequently there are no adenoids, and, furthermore, in at least $20 \mathrm{per}$ cent. of cases of adenoids of the second diffuse type operation fails to cure the discharge and obstruction. This heterodox view, that nasal stenosis is the essential lesion, is borne out by the results of treatment.

The object of treatment must be to restore the patency of the nasal passages. Naturally one must deal with any cause of obstruction which one discovers: Adenoids must be removed, and I agree with Dr. Dan McKenzie that the adenotome is a more accurate and scientific instrument than the curétte.

Enlarged turbinals must be reduced by partial removal, and special attention paid to enlarged " posterior ends." I have never discovered posterior ends in children under the age of 6 , though they are not at all uncommon in older children.

As regards deviation of the nasal septum, this cause of obstruction is, in my experience, rather rare in childhood, apart from deviation directly due to injury. The deviation involves, as a rule, only the cartilage and not the bone. There need be no hesitation in performing a limited submucous resection, and I have never seen deformity follow operation when care has been taken to remove only sufficient cartilage to relieve the nasal stenosis.

There remains, -however, the large group of patients who show no obvious cause of the nasal catarrh, althougl the entire nasal cavity may be smaller and less developed than it should be. What can be done for those little patients?

Regular removal of the muco-purulent secretion is the first essential. In babies a simple suction apparatus (mouche bébé) is useful. Older children must be taught how to blow the nose (by the open method, not by grasping the nose), although this is a difficult task for teacher and pupil. Local applications may follow this cleansing regime, and one of the best is the French preparation gomenol, which is used in 5 per cent. solution in olive-oil, and dropped into the nose with a pipette.

As regards internal treatment, the "catarrhal" child derives benefit from sodium bicarbonate in large doses. 1 cannot explain this action, but the free use of such an alkali, by the mouth, is of great value in middle-ear suppuration, as well as in chronic rhinitis.

"The so-called "catarrhal" and thickened mucous membrane may often be restored to normal by the method of "diastolization" introduced by Gautier in France five years ago, but only recently adopted in this country. The procedure is virtually a means of massaging the nasal mucosa by the expansion and dilatation of a thin hollow rubber bougie, connected to an otomasseur.

The dental condition is of some importance. When the upper dental arch is narrow and the teeth crowded it may be resirable to fit an expanding denture composed of two halves. This aspect of nasal obstruction in children has perhaps been unduly stressed by our dental colleagues, but it certainly deserves attention in selected cases.

Most important, however, is the restoration of the perverted respiratory function by systematic re-education. Breathing exercises are indispensable, but in carrying out the exercises, expiration, as well as inspiration, must bo nasal. It is essential to insist on this, as one often finds that the teacher encourages children to take a deep breatl through the nose and then let the air escape by the mouth. This is, of course, quite an unnatural method of respiration. It is even worse than the method sometimes ado -ied after adenoid removal-namely, no instructions and no exercises at all!

By such methods, conducted with diligence and care, the patency of the nasal airway may be gradually restored, and as the patient loses his mouth-breathing habit and acquires the normal manner of respiration by the nasal route, the nasal discharge will disappear. 